\title{
Long-range Order in One-dimensional Spinless Fermi Gas with Attractive Dipole-Dipole Interaction
}

\author{
Zhongbo Yan ${ }^{1}$, Liang Chen $^{2}$, and Shaolong Wan $^{1 *}$ \\ ${ }^{1}$ Institute for Theoretical Physics and Department of Modern Physics \\ University of Science and Technology of China, Hefei, 230026, P. R. China \\ ${ }^{2}$ Beijing Computational Science Research Center, Beijing, 100084, P. R. China
}

(Dated: September 24, 2018)

\begin{abstract}
One-dimensional spinless Fermi gas with attractive dipole-dipole interaction is investigated. Results obtained show when the interaction is weak, the excitation spectrum is linear and the superconducting correlation function decays as power law, indicating the validity of the Tomonaga-Luttinger (TL) liquid picture. However, when the interaction reaches a critical value, the excitation spectrum is nonlinear and the superconducting correlation function keeps finite for infinity separation, indicating real long-range order established and the breakdown of the TL liquid picture. We prove that the existence of long-range order is not in contradiction with the Hohenberg theorem and show that this system is related to the Kitaev toy model, therefore, it has potential applications for the future topological quantum computation.
\end{abstract}

PACS numbers: 71.10.Ca, 71.10.Pm, 71.45.Lr, 74.20.Rp

Introduction. - The Tomonaga-Luttinger (TL) liquid theory [1-3] can well describe a lot of one-dimensional physics systems $[4,5]$, such as the edge state of a topological nontrivial system, quantum wires, organic conductors, etc. For one-dimensional homogeneous spinless fermions, if the fermions are neutral (cold atomic system), usually the system is described by a free Fermi gas model, since the two-body collisions are negligible due to the Pauli principle. If the fermions are charged, like fully polarized electrons, they feel a long-range Coulumb repulsive interaction, and the repulsive interaction drives the system far away from the free Fermi gas to the quasiWigner crystal phase [6].

In this paper, we consider one-dimensional spinless fermions with dipole-dipole interaction in a cold atomic system. The dipolar interaction has recently arisen a lot of interests in cold atomic field [7-9] due to its longrange and anisotropic character. The long-range and anisotropic character can induce many peculiar effects in both bosonic and fermionic systems. For bosons, for example, the dipole-dipole interaction can induce the excitation spectrum of a Bose-Einstein condensation to exhibit interesting roton structure [10-12]. For fermions, under different conditions the dipole-dipole interaction can induce numerous phases of interest, such as p-wave superfluid [13, 14], ferronematic phase [15], liquid crystal phase [16] and other phases.

For neutral spinless fermions in cold atomic system, because the collisions between fermions are negligible, the dipole-dipole interaction is the only familiar and available interaction that can be used to drive the system away from the free Fermi gas. Before making any progress, we should notice that the dipole-dipole interaction in one dimension is no longer long-range but finite range. As a result, if the dipoles are aligned to the direction perpendicular to the system which indicates that the interaction is repulsive, no critical behavior like quasi-Wigner crystal phase emerges and the system is well described by the TL liquid theory for all interaction strength $[17,18]$. The purpose of the present paper is to investigate the effects of the attractive dipole-dipole interaction which is induced by aligning the dipoles along the one-dimensional system. The main conclusion we have obtained by bosonization technique is that the finite-range attractive dipoledipole interaction, when its strength reaches a critical value where TL liquid theory reaches the critical point of validity, establishes a phase (superfluid) with real longrange order, instead of quasi-long-range order which is power-law decay.

Model. - For one-dimensional homogeneous spinless fermions with attractive dipole-dipole interaction, the Hamiltonian has the form (here setting $\hbar=1$ )

$H=\sum_{k ; r= \pm} v_{F}\left(r k-k_{F}\right) c_{r, k}^{\dagger} c_{r, k}+\frac{1}{2 L} \sum_{q} V(q) \rho(q) \rho(-q),(1)$

where $r=+$ for right going particles and $r=-$ for left going particles. $L$ is the length of the system. $\rho(q)=$ $\sum_{r, k} c_{r, k+q}^{\dagger} c_{r, k}$ is the density fluctuation operator. We also define $\rho_{r}(q)=\sum_{k} c_{r, k+q}^{\dagger} c_{r, k}$ for convenience. $V(q)$ is the Fourier form of the dipole-dipole interaction

$$
V(x)=-\frac{C_{d d}}{\left[x^{2}+d^{2}\right]^{\frac{3}{2}}},
$$

and takes the form

$$
V(q)=-\frac{2 C_{d d}}{d^{2}}|q d| K_{1}(q d),
$$

where $K_{1}$ denotes the 1-st order modified Bessel function, $C_{d d}=\tilde{d}^{2} / 2 \pi \epsilon_{0}$ for fermions with electric dipole moment $\tilde{d}$ or $C_{d d}=\mu_{0} \mu^{2} / 2 \pi$ for fermions with magnetic dipole moment $\mu$. For the dipole-dipole interaction, we followed 
Ref.[6] and introduced a cut off $d$ to avoid the divergence at $r=0$. The cut off $d=\lambda\left(l / l_{\perp}\right) l_{\perp}$, where $\lambda\left(l / l_{\perp}\right)$ is a constant whose value is determined by the ratio $l / l_{\perp}$, $l$ is the length of the dipole and $l_{\perp}=\sqrt{\hbar / m \omega_{\perp}}$ is the radial confinement length. In fact, the simple interaction form (2) has maintained the essential properties of the dipole-dipole interaction both for long distance and short distance. This can be easily seen if we deduce the dipole-dipole interaction from the microscopic structure of the dipole. The moment of the dipole is defined as $\tilde{d}=q l$, where $q$ is the polarized charge and $l$ is the length as mentioned above. As charges interact with each other according to the Coulomb principle, the interaction between two dipoles is given as

$$
V(x)=-\frac{q^{2}}{4 \pi \epsilon_{0}}\left(\frac{1}{x}+\frac{1}{x+2 l}-\frac{2}{x+l}\right),
$$

where $x$ is the distance from the left dipole's tail to the right dipole's head in the one-dimensional configuration. Such an interaction form also divergent at $x=0$. To avoid the divergence, we introduce the transverse confinement $l_{\perp}$ as a cut off and give

$$
\begin{aligned}
V(x)=-\frac{q^{2}}{4 \pi \epsilon_{0}}( & \frac{1}{\left(x^{2}+l_{\perp}^{2}\right)^{1 / 2}}+\frac{1}{\left((x+2 l)^{2}+l_{\perp}^{2}\right)^{1 / 2}} \\
& \left.-\frac{2}{\left((x+l)^{2}+l_{\perp}^{2}\right)^{1 / 2}}\right) .
\end{aligned}
$$

When $x>>d, l$, by using a Taylor expansion, we obtain $V(x)=-\frac{C_{d d}}{x^{3}}$. When $x \rightarrow 0$ and $l_{\perp}<<l, V(x)=$ $-\frac{C_{d d}}{2 l_{\perp} l^{2}}\left(1-\frac{l_{\perp}}{l}\right)$. However, when $x \rightarrow 0$ and $l_{\perp}>>l$, $V(x)=\frac{C_{d d}}{l_{\perp}^{3}}$, which is positive. This indicates that the interaction form (2) is valid for short distance only when the system is really very close to be one-dimensional, i.e., $l_{\perp}$ is the same order of $l$. In this paper, we set $l_{\perp}=l$ for simplicity, then $V(x=0)=-\frac{C_{d d}}{d^{3}}$, with $\lambda\left(l / l_{\perp}\right) \approx 60^{\frac{1}{3}}$. Based on the analysis above, the justification of using (2) to substitute the dipole-dipole interaction is evident.

In fact, we more care about the Fourier form of the interaction, because it determines all quantities we care about. From (3), we can see that both $C_{d d}$ and $d$ can be easily tuned, therefore the interaction can have a wide range. Furthermore, to guarantee that the system is effectively one-dimensional, the density $n$ should satisfy the condition: $n<1 / l_{\perp}$.

We rewrite the Hamiltonian (1) into the g-ology description

$$
\begin{aligned}
H= & \frac{\pi v_{F}}{L} \sum_{p \neq 0} \sum_{r= \pm}: \rho_{r}(p) \rho_{r}(-p): \\
& +\frac{2}{L} \sum_{p} g_{2}(p) \rho_{+}(p) \rho_{-}(-p) \\
& +\frac{1}{L} \sum_{p, r= \pm} g_{4}(p): \rho_{r}(p) \rho_{r}(-p):
\end{aligned}
$$

where $g_{2}(p)=g_{4}(p)=V(p) / 2$. Here we have neglected some constant terms which do not affect the physics we consider.

Following standard procedures in the bosonization, the Hamiltonian (4) can be easily diagonalized. The diagonalized Hamiltonian $H_{D}$ takes the form

$$
H_{D}=\frac{\pi}{L} \sum_{p \neq 0} \sum_{r= \pm} v_{\rho}(p): \rho_{r}(p) \rho_{r}(-p): .
$$

Here we also only neglect the constant terms and only keep the terms related to the physics we care about. In Hamiltonian (5), $v_{\rho}(p)=v_{F} / K_{\rho}(p)=$ $v_{F} \sqrt{1+V(p) / \hbar \pi v_{F}}$ (based on dimension analysis, here we have reintroduced $\hbar$ for following use). $K_{\rho}(p)=$ $1 / \sqrt{1+V(p) / \hbar \pi v_{F}}$ is the stiffness which determines quantities like different kinds of correlation functions.

In one dimension, it's known that the $1 / r$ long-range Coulomb repulsive interaction enhances the $4 k_{F}$ charge density correlations for spinful electrons, and drives the system to a Wigner crystal which shows a critical behavior quite different from the ordinary TL liquid [6]. Such a Wigner crystal phase is due to the fact that the Fourier form of the $1 / r$ long-range repulsive interaction $V_{e}(q)$ is logarithmic divergent for $q \rightarrow 0$. A divergent $V_{e}(q=0)$ means $K_{\rho}(p=0)=0$ (for small but non-zero momentum, $K_{\rho}(p)$ keeps small but finite). Here a fact that we should notice is that the interaction is long-range, instead of a short one which can usually be treated as a delta interaction, the dependence on momentum of $K_{\rho}$ is important and we can no longer just use $K_{\rho}(p=0)$ to substitute the whole $K_{\rho}(q)$ when we calculate quantities like the correlation functions.

For the system with delta-form attractive interaction, it is know that when the interaction increases, the fluctuation of superconducting order is enhanced, however, when the interaction is strong enough to make $K_{\rho} \rightarrow \infty$, instead of real long-range superconducting order established, the TL liquid picture breaks down, as the compressibility $\kappa=2 K_{\rho} / n^{2} \pi v_{\rho}$ is divergent and the system is phase separated [19, 20]. For attractive dipolar-dipolar interaction, the interaction is finite-range and the momentum dependence of the Fourier form needs to be considered. As $K_{\rho}(p=0)^{-1}$ decreases with increasing interaction, it will touch zero when the interaction reaches a critical value. However, as the interaction is finiterange, like the long-range Coulomb interaction, now we can no longer just use $K_{\rho}(p=0)^{-1}$ to substitute the whole $K_{\rho}(q)^{-1}$ when we calculate the superconducting correlation function. Because of the momentum dependence, the relation $\kappa=2 K_{\rho} / n^{2} \pi v_{\rho}$ is no longer justified, in other words, $K_{\rho}(p=0)^{-1}=0$ does not indicate the compressibility is divergent and the system will be phase separated.

In statistical mechanics, the compressibility is defined as $\kappa=n^{-2}(\partial n / \partial \mu)_{T}$. The divergent of compressibility 
means that changing the number of the particles, the chemical potential does not change, i.e., $\Delta \mu=E(N+$ $1)+E(N-1)-2 E(N)=0$. For a TL liquid with system length $L, \Delta \mu$ should be approximately proportional to $v_{\rho, \mathrm{N}+1}\left(q_{\mathrm{N}+1}\right) q_{\mathrm{N}+1}-v_{\rho, \mathrm{N}}\left(q_{\mathrm{N}}\right) q_{\mathrm{N}}$, where $q_{\mathrm{N}+1}=q_{\mathrm{N}}+\frac{\pi}{L}$. This agrees with the fact that, more generally, a vanishing $v_{\rho}$, instead of an infinity $K_{\rho}$, corresponds to the instability of phase separation [20]. However, for attractive dipoledipole interaction, when the interaction reaches the critical value, the velocity only vanishes at $q=0$ (where the bosonic operator is also not defined), and furthermore, here $v_{\rho, \mathrm{N}}$ is anti-proportional to $N$ (see following). This indicates $\Delta \mu$ may be very small, but not zero. Therefore, when the interaction reaches the critical value, the system should not be phase-separated.

In bosonization language, the single particle fermionic operator is given as

$$
\psi_{r}(x)=\lim _{\alpha \rightarrow 0} \frac{U_{r}}{\sqrt{2 \pi \alpha}} e^{i r k_{F} x} e^{-i[r \phi(x)-\theta(x)]},
$$

where $U_{r}$ is known as Klein factor. For repulsive interaction, $K_{\rho}(p)<1$, the system is inclined to order in charge density wave and the charge correlation function $<\rho(x) \rho(0)>$ which is dominated by the quantity given as

$$
\begin{aligned}
& \frac{2}{(2 \pi \alpha)^{2}} \cos \left(2 k_{F} x\right) e^{-2<\left[\phi_{\rho}(x)-\phi_{\rho}(0)\right]^{2}>} \\
& =\frac{2}{(2 \pi \alpha)^{2}} \cos \left(2 k_{F} x\right) e^{-2 \int_{0}^{\infty} \frac{d p}{p} K_{\rho}(p)(1-\cos p x)}
\end{aligned}
$$

is mainly considered. From (7), it is easy to see if we directly substitute $K_{\rho}(p)$ as $K_{\rho}(p=0)$ which takes value zero for the long-range repulsive Coulomb interaction, the correlation function will exhibit perfect crystalline order, which is a wrong conclusion as shown in Ref.[6]. This gives us a concrete example that we can not directly use $K_{\rho}(p=0)$ to substitute $K_{\rho}(p)$ when the interaction is strong enough to induce a vanishing $K_{\rho}(p=0)$, or else wrong conclusion may be obtained.

For attractive interaction, $K_{\rho}(p)>1$, the system is inclined to order in superconductivity and what we care about is the superconducting correlation function which is mainly determined by the quantity given as

$$
\begin{aligned}
& \frac{1}{(\pi \alpha)^{2}} e^{-2<\left[\theta_{\rho}(x)-\theta_{\rho}(0)\right]^{2}>} \\
& =\frac{1}{(\pi \alpha)^{2}} e^{-2 \int_{0}^{\infty} \frac{d p}{p} K_{\rho}^{-1}(p)(1-\cos p x)} .
\end{aligned}
$$

The similarity of the form between $<\left[\phi_{\rho}(x)-\phi_{\rho}(0)\right]^{2}>$ and $<\left[\theta_{\rho}(x)-\theta_{\rho}(0)\right]^{2}>$ is just the basis for our expectation that like the long-range repulsive Coulomb interaction diving the charge correlation function to exhibit a quasi-Wigner crystal phase [6], the finite-range attractive dipole-dipole interaction may also drive the superconducting correlation functions to exhibit some critical behavior when $K_{\rho}^{-1}(p=0)=0$ (as mentioned above, for non-zero momentum, $K_{\rho}^{-1}(p)$ needs to be small but finite to against the phase-separation instability). From $(8)$, it is also easy to see we can not directly substitute $K_{\rho}^{-1}(p)$ as $K_{\rho}^{-1}(p=0)$ when $K_{\rho}^{-1}(p=0)$ takes the critical value zero, or else the superconducting correlation function does not depend on distance, which is obviously unphysical. For delta-form attractive interaction, as $K_{\rho}^{-1}(p)=K_{\rho}^{-1}(p=0)$, when $K_{\rho} \rightarrow \infty$, the result of the superconducting correlation function is unphysical, this also indicates that the TL liquid picture is broken down, and the system is phase-separated.

When $K_{\rho}^{-1}(p=0)=0$, we find $V(p) / \hbar \pi v_{F}=$ $-|p d| K_{1}(p d)$ or $2 C_{d d} / \hbar \pi v_{F} d^{2}=1$. If we define a dimensionless parameter $\gamma=2 C_{d d} / \hbar \pi v_{F} d^{2}$, we find, for $\gamma<1$, the dispersion takes the form $\omega_{\rho}(p) \sim p$ in the long-wavelength limit, which indicates a TL liquid and for $\gamma=1, \omega_{\rho}(p) \sim\left|p^{4} \log p\right|^{\frac{1}{2}}$, the dispersion is still gapless but no longer linear, indicating that different physics may emerge. By a direct calculating, we find, for $\gamma<1$

$$
\begin{aligned}
& \frac{1}{(\pi \alpha)^{2}} e^{-2<\left[\theta_{\rho}(x)-\theta_{\rho}(0)\right]^{2}>} \\
& =\frac{1}{(\pi \alpha)^{2}} e^{-2 \int_{0}^{1 / \alpha} \frac{d p}{p} K_{\rho}^{-1}(p)(1-\cos p x)} \\
& \simeq \frac{1}{(\pi \alpha)^{2}} e^{-2 \int_{0}^{1 / d} \frac{d p}{p} K_{\rho}^{-1}(p=0)(1-\cos p x)-2 \ln (d / \alpha)} \\
& =\frac{1}{(\pi d)^{2}}\left(\frac{d}{x}\right)^{2 \sqrt{1-\gamma}} .
\end{aligned}
$$

Here we substitute $K_{\rho}^{-1}(p)$ as $K_{\rho}^{-1}(p=0)$, since when $K_{\rho}^{-1}(p=0) \neq 0$, such a substitution does not affect the quality behavior (power-law decay) of the superconducting correlation function. For $\gamma=1$, after taking the same procedures, we obtain

$$
\begin{aligned}
& \frac{1}{(\pi \alpha)^{2}} e^{-2<\left[\theta_{\rho}(x)-\theta_{\rho}(0)\right]^{2}>} \\
& =\frac{1}{(\pi d)^{2}} e^{-2 \int_{0}^{1 / d} \frac{d p}{p} K_{\rho}^{-1}(p)(1-\cos p x)} \\
& >\frac{1}{(\pi d)^{2}} e^{-4 \int_{0}^{\frac{1}{d}} \frac{d p}{p} \sqrt{1-|p d| K_{1}(p d)}} \\
& =\frac{1}{(\pi d)^{2}} e^{-4 \int_{0}^{1} \frac{d y}{y} \sqrt{1-y K_{1}(y)}} \\
& =\frac{1}{(\pi d)^{2}} e^{-3.56}
\end{aligned}
$$

here we have introduced a cutoff $1 / d$ for the momentum to avoid nonphysical divergence. The results above indicates when $\gamma<1$, the superconducting correlation function exhibits a familiar power law decay and vanishes for $x \rightarrow \infty$, agreeing with our previous arguments based on the dispersion that the system falls into the TL liquid for $\gamma<1$. While $\gamma=1$, the superconducting correlation function keeps finite for $x \rightarrow \infty$, indicating a real longrange order established and simultaneously the TL liquid 
picture reaches the critical point of validity. This is our main result in this paper.

The existence of long-range superconducting order here does not contradict with the Hohenberg theorem which strictly roles out Bose-Einstein condensation and superconductivity at finite temperature in one and two dimension [21]. We can prove this by following the procedures used in Ref.[21]. Before giving the details of proof, we should notice the fact that here the Cooper pair is composed of two spinless fermions, the anomalous average $\left\langle\psi(x) \psi\left(x^{\prime}\right)>\right.$ is the quantity to characterize the long-range order. After noticing this difference, we follow the procedures applied in Ref.[21]. First, we similarly introduce the order parameter

$$
\Delta(x)=\int d x^{\prime} s\left(x-x^{\prime}\right)<\psi(x) \psi\left(x^{\prime}\right)>,
$$

where $s(x)$ is a smearing function. By Fourier transformation, we have

$$
\Delta(k)=\Omega^{-1} \sum_{q} S(q)<c_{k-q} c_{q}>.
$$

We assume the smearing function has inversion symmetry, therefore, $S(q)=S(-q)$. As a result, $\Delta(0)=0$.

We apply the Bogoliubov inequality to the operators

$$
\begin{aligned}
& A_{k}=i(\partial / \partial t) \rho_{-k}(t) \\
& B_{k}=\sum_{q} S(q) c_{k-q} c_{q} .
\end{aligned}
$$

The fermion commutation rules yield for $k \neq 0$

$$
\begin{aligned}
\frac{1}{\Omega}<\left[B_{k}, \rho_{-k}\right]> & =\frac{1}{\Omega} \sum_{q}[S(q)+S(k-q)]<c_{q} c_{-q}> \\
& =\Delta(0)+\eta(k)=\eta(k),
\end{aligned}
$$

it's easy to see that $\eta(k)$ is antisymmetric, i.e., $\eta(k)=$ $-\eta(-k)$, which implies $\eta(k) \propto k^{2 n+1}(\mathrm{n}=0,1, \ldots)$ for $k \rightarrow$ 0 . Now the Bogoliubov inequality is given as

$$
C_{B, B^{\dagger}}(k) \geq 2 T \frac{|\eta(k)|^{2}}{(n / m) k^{2}} .
$$

The concrete form of $C_{B, B^{\dagger}}(k)$ is given as

$$
\begin{aligned}
C_{B, B^{\dagger}}(k)= & \Omega^{-1}<\left\{\sum_{p} S(p) c_{k-p} c_{p}, \sum_{q} S(q) c_{q}^{\dagger} c_{k-q}^{\dagger}\right\}> \\
= & 2 \Omega^{-1} \sum_{p, q} S(p) S(q)<c_{q}^{\dagger} c_{k-q}^{\dagger} c_{k-p} c_{p}> \\
& -\Omega^{-1} \sum_{q}[S(q)-S(k-q)]^{2}<c_{q}^{\dagger} c_{q}> \\
& +\Omega^{-1} \sum_{q} S(q)(S(q)-S(k-q)) \\
= & F(k)+R(k) .
\end{aligned}
$$

where $F(k)$ denotes the first term in the second line, and $R(k)$ denotes the rest of terms and is regular at small $k$. The Fourier transform of $F(k)$ is given as

$$
\begin{aligned}
f\left(x_{2}-x_{1}\right) \equiv & \iint d x^{\prime} d x^{\prime \prime} s\left(x_{1}-x^{\prime}\right) s\left(x_{2}-x^{\prime \prime}\right) \\
& \times<\psi^{\dagger}\left(x^{\prime}\right) \psi^{\dagger}\left(x_{1}\right) \psi\left(x_{2}\right) \psi\left(x^{\prime \prime}\right)>
\end{aligned}
$$

By using the conclusion of Ref.[21], we obtain

$$
f(0) \leq 2\left[\int d x^{\prime} s\left(x_{1}-x^{\prime}\right)\left\{<\rho\left(x_{1}\right) \rho\left(x^{\prime}\right)>\right\}\right]^{2} .
$$

As pointed by Hohenberg, $f(0)$ is finite since the density correlation function may not have any singularities which are not integrable. Combining Eqs.(14), (15) and (17), we finally have

$$
\begin{gathered}
F(k) \geq 2 T \frac{|\eta(k)|^{2}}{(n / m) k^{2}}-R(k), \\
\Omega^{-1} \sum_{k \neq 0} F(k)<f(0)<\infty .
\end{gathered}
$$

In Ref.[21], since $\Delta+\eta(k) \rightarrow 2 \Delta$ and $\Delta$ is a non-zero constant, Eqs.(18) and (19) are in contraction in one and two dimension for $T \neq 0$ and for infinite volume. However, for spinless fermions, $\eta(k) \sim k^{2 n+1}(\mathrm{n}=0,1, \ldots)$ for $k \rightarrow 0$, as a result, the infrared divergence is removed and $F(k)$ is finite. The contraction appearing in Ref.[21] disappears and Eq.(18) and Eq.(19) are now self-consistent. $\left\langle c_{q} c_{-q}\right\rangle$ also no longer needs to vanish for any $q$. From this, we have proved that the existence of long-range superconducting order for spinless fermions with attractive dipolar interaction does not have any contradiction with the Hohenberg theorem.

For spinful fermions, however, there is no such critical behavior. This is due to the fact that spin and charge degrees of spinful fermions will separate in one dimension, which is illustrated by $K_{\rho}(p) \neq K_{\sigma}(p)$. Furthermore, it is not hard to find when $K_{\rho}(p) \rightarrow \infty, K_{\sigma}(p)$ and $K_{\sigma}^{-1}(p)$ keep finite. As a result, the superconducting correlation functions proportional to $e^{-\int_{0}^{\infty} \frac{d p}{p}\left(K_{\rho}^{-1}(p)+K_{\sigma}(p)\right)(1-\cos p x)}$ or $e^{-\int_{0}^{\infty} \frac{d p}{p}\left(K_{\rho}^{-1}(p)+K_{\sigma}^{-1}(p)\right)(1-\cos p x)}$ always give a power law and therefore always vanishes for $x \rightarrow \infty$. No real long-range order exists at least when the attractive interaction is not strong enough to go beyond the Luttinger liquid picture.

Related to the Kitaev toy model. - As the dipole-dipole interaction is not truly long-range in one dimension, the collisions between spinless fermions is dominated by $p$ wave collisions at low temperature. Therefore, based on the arguments in the previous section, when real longrange order is established for sufficiently strong attractive interaction, the system is a real $p$-wave superfluid and it is a realization of the Kitaev toy model [22]. This suggests such a system has potential applications for the future topological quantum computation. 
Experiments.-To observe the critical behavior, we already know that the interaction should reach the critical value, i.e., $\gamma_{c}=2 C_{d d} / \hbar \pi v_{F} d^{2}=$ $\left(2 \tilde{d}^{2} m\right) /\left(2 \pi^{3} \epsilon_{0} \hbar^{2} \lambda^{2} l_{\perp}^{2} n\right)=1$. Assuming $n=0.25 / l_{\perp}$, then for polar molecules composed of alkali atoms [23], $m \sim 100 u\left(1 u=1.66 \times 10^{-27} \mathrm{~kg}\right), l_{\perp}=l \sim 10 \AA$ (corresponds to $\omega_{\perp} \sim 600 \mathrm{MHz}$ ), we obtain the critical $\tilde{d}_{c}=\left(\pi^{3} \epsilon_{0} \hbar^{2} \lambda^{2} l_{\perp}^{2} n / m\right)^{1 / 2}=0.075$ Debye, which is within the current experimental ability. From the expression of $d_{c}$, it's easy to see that a lower density $n$ corresponds to a lower $d_{c}$, and a lower $d_{c}$ corresponds to a weaker interaction, therefore, the three-body loss is not worthy of worry.

In future, once the polar molecules can be cooled to quantum degeneracy, the system proposed in this paper is realizable under current experimental conditions and therefore its applications for topological quantum computation is worth expecting.

Conclusion.-Using bosonization technique, both the excitation spectrum and the superconducting correlation function of one-dimensional spinless fermi gas with attractive dipolar interaction are obtained. The superconducting correlation function exhibits a critical behavior that when the interaction reaches the critical value, i.e. $\gamma=1$, it keeps finite for infinity separation, indicating real long-range order established. The existence of this long-range order has no contradiction with the Hohenberg theorem and makes the system to be a realization of Kitaev toy model.

Acknowledgments. - This work is supported by NSFC. Grant No.11275180.

\footnotetext{
*slwan@ustc.edu.cn
}

[1] S. Tomonaga, Prog. Theor. Phys. 5, 544 (1950).

[2] J. M. Luttinger, J. Math. Phys. 4, 1154 (1963)

[3] F. D. M. Haldane, J. Phys. C 14, 2585 (1981).

[4] A. O. Gogolin, A. A. Nersesyan, A. M. Tsvelik, Bosonization and Strongly Correlated Systems, Cambridge University Press (1998).

[5] T. Giamarchi, Quantum Physics in One Dimension, Clarendon Press, Oxford (2003).

[6] H. J. Schulz, Phys. Rev. Lett. 71, 1864 (1993).

[7] L. You and M. Marinescu, Phys. Rev. A 60, 2324 (1999).

[8] M. A. Baranov, Physics Reports 464, 71 (2008).

[9] T. Lahaye, C. Menotti, L. Santos, M. Lewenstein, and T. Pfau, Rep. Prog. Phys. 72, 126401 (2009)

[10] D. H. J. ODell, S. Giovanazzi and G. Kurizki, Phys. Rev. Lett. 90, 110402 (2003);

[11] L. Santos, G. V. Shlyapikov, M. Lewenstein, Phys. Rev. Lett. 90, 250403 (2003).

[12] S. Ronen, D. C. E. Bortolotti, J. L. Bohn, Phys. Rev. Lett. 98, 030406 (2007).

[13] M. A. Baranov, M. S. Mar'enko, Val. S. Rychkov, G. V. Shlyapnikov, Phys. Rev. A 66,013606 (2002).

[14] G. M. Bruun, and E. Taylor, Phys. Rev. Lett. 101, 245301 (2008).

[15] B. M. Fregoso and E. Fradkin, Phys. Rev. Lett. 103, 205301 (2009).

[16] Chungwei Lin, Erhai Zhao, and W. Vincent Liu, Phys. Rev. B 81,045115 (2010).

[17] Y. Tsukamoto and N. Kawakami, J. Phys. Soc. Jpn. 69, 149 (2000).

[18] H. Inoue, and K. Nomura, J. Phys. A: Math. Gen. 39, 2161 (2006).

[19] M. Nakamura and K. Nomura, Phys. Rev. B 56, 12840 (1997).

[20] D. C. Cabra and J. E. Drut, J. Phys. Cond. Matt. 15 14445 (2003).

[21] P. C. Hohenberg, Phys. Rev. 158, 383 (1967).

[22] A. Yu. Kitaev, Phys.-Usp. 44, 131 (2001).

[23] K.-K. Ni, et al, Science 322, 231 (2008). 